\title{
Variações sazonais nas concentrações de pigmentos e nutrientes em folhas de espécies de cerrado com diferentes estratégias fenológicas
}

\author{
ANA PAULA F. CARVALHO', MERCEDES M.C. BUSTAMANTE ${ }^{1,3}$, \\ ALESSANDRA R. KOZOVITS ${ }^{1}$ e GREGORY P. ASNER ${ }^{2}$
}

(recebido: 18 de agosto de 2005; aceito: 30 de setembro de 2006)

\begin{abstract}
Seasonal variations in leaf pigments and nutrients in "cerrado" species with different phenological patterns). Several studies have been trying to establish relationships between environmental conditions and leaf traits. In the present study, concentrations of pigments (chlorophylls a, b, carotenoids and anthocyanins), N and P and specific leaf area (SLA) were measured in leaves of six "cerrado" species (three deciduous and three evergreen species) in two cerrado stricto sensu sites with differences in canopy cover and in different periods: at the beginning of the dry season (June), end of dry season (September) and beginning of the rainy season (November). Nutrient concentrations differed significantly as a function of phenology and seasonality while pigments were more sensitive to changes in vegetation structure. The highest chlorophyll $a$ and $b$ concentrations were measured at the beginning of the dry season (June) in comparison to the end of the dry season (September) in both sites. In June, chlorophyll $a$ and $b$ concentrations were higher in the denser cerrado while the chlorophyll $a / b$ ratio was lower. Total chlorophyll/carotenoids ratio was also significantly higher in the denser cerrado at the beginning of the dry season due to the higher chlorophyll concentration. Deciduous species showed higher SLA mean values than evergreen species, in dry and wet season but higher $\mathrm{N}$ and $\mathrm{P}$ concentrations were found in the evergreen species. In both phenological groups, $\mathrm{N}$ and $\mathrm{P}$ concentrations and the N:P ratios were higher in the wet season (November) than in the dry season (June).
\end{abstract}

Key words - chlorophylls, phenology, savanna, specific leaf area

RESUMO - (Variações sazonais nas concentrações de pigmentos e nutrientes em folhas de espécies de cerrado com diferentes estratégias fenológicas). Vários estudos têm procurado estabelecer relações entre as condições ambientais e as propriedades foliares. Neste trabalho foram medidas as concentrações de pigmentos (clorofilas $a, b$, carotenóides e antocianinas), $\mathrm{N}$ e $\mathrm{P} \mathrm{e}$ área foliar específica (AFE) em folhas de seis espécies de cerrado (sendo três decíduas e três sempre-verdes) em dois sítios de cerrado stricto sensu com diferenças de cobertura arbustiva-arbórea e em diferentes períodos: início do período seco (junho), fim da seca (setembro) e início das chuvas (novembro). As concentrações de nutrientes variaram mais em função da fenologia e da sazonalidade do que em relação às diferenças estruturais nos sítios, ao contrário do que foi observado para os pigmentos foliares. Os maiores valores de concentração de clorofilas $a$ e $b$ foram encontrados no início (junho) em relação ao final da seca (setembro) nas duas áreas. Em junho, as concentrações de clorofilas $a$ e $b$ foram maiores na área mais densa enquanto a razão clorofila $a / b$ foi menor. A razão clorofila total/carotenóides também foi significativamente maior no cerrado fechado em relação ao aberto nesse período devido às maiores concentrações de clorofilas no primeiro sítio. Espécies decíduas apresentaram maiores médias de AFE em relação às sempre-verdes, tanto na estação seca como na chuvosa, mas concentrações mais elevadas de $\mathrm{N}$ e $\mathrm{P}$ foram encontradas em espécies sempre-verdes em relação às decíduas. Nos dois grupos fenológicos, as concentrações foliares de N e P e a razão N:P foram maiores na estação chuvosa (novembro) em relação à estação seca (junho).

Palavras-chave - área foliar específica, cerrado, clorofilas, fenologia

\section{Introdução}

Características de plantas e suas relações com fatores ambientais têm sido descritas com o objetivo de promover comparações em nível global (Grime et al. 1997, Garnier et al. 2001). Características foliares são

1. Universidade de Brasília, Departamento de Ecologia, 70910-970 Brasília, Brasil.

2. Carnegie Institution of Washington, Department of Global Ecology, 260 Panama, St. Stanford, CA, USA.

3. Autor para correspondência: mercedes@unb.br freqüentemente citadas como principais indicadores no relacionamento do uso de recursos pelas plantas, biomassa e funcionamento do ecossistema (Craine et al. 2001) por serem de fácil quantificação e estarem fortemente relacionadas à fisiologia das plantas (Garnier et al. 2001). As características foliares são geralmente medidas no auge da estação de crescimento quando as condições ambientais favorecem a assimilação de carbono e há abundância de folhas expandidas e saudáveis. Muitos conjuntos de dados encontram-se disponíveis para o estudo das características foliares e suas inter-relações em ambientes temperados 
(Niinemets 2001). No entanto, há grande deficiência de dados para ambientes sazonais de florestas tropicais secas e savanas (Prior et al. 2004).

A vegetação das savanas é composta por uma camada contínua de gramíneas e outra descontínua de árvores. Os maiores limitantes de produtividade desses ecossistemas são a baixa disponibilidade de água na estação seca, a baixa fertilidade dos solos e o fogo (Prior et al. 2003). Entre as savanas sazonais incluem-se os cerrados do Brasil Central, onde a camada arbórea é composta por uma mistura de espécies sempre-verdes e decíduas. O entendimento da coexistência de espécies com diferentes estratégias fenológicas demanda ainda maiores estudos. Franco et al. (2005) estudaram diferenças entre as espécies decíduas e sempre-verdes de cerrado quanto à eficiência no uso da água no auge da estação seca. As espécies decíduas apresentaram maiores valores de $A_{\text {mass }}$ (assimilação máxima de $\mathrm{CO}_{2}$ por massa), $\mathrm{N} \mathrm{e}$ $\mathrm{P}$ foliares e maior eficiência no uso da água no fim da estação seca com a emissão de folhas novas e com menor custo de construção das folhas (maior área foliar específica - AFE) suportando a hipótese de que folhas de vida mais longa possuem maior custo de construção e que ocorre um maior investimento em $\mathrm{N}$ em folhas de vida mais curta, o que possibilita maiores valores de $A_{\max }$ (assimilação máxima de $\mathrm{CO}_{2}$ ) com um tempo de retorno menor.

Apesar da relevância dos pigmentos fotossintéticos para o funcionamento de folhas, ainda não há trabalhos que relacionem as variações sazonais em suas concentrações em espécies de cerrado com diferentes padrões fenológicos. Nesse sentido, o presente trabalho avalia a variação sazonal nas concentrações foliares de clorofilas, carotenóides, antocianinas, nitrogênio, fósforo e área foliar específica em espécies de cerrado crescendo em dois sítios com diferentes densidades de lenhosas.

\section{Material e métodos}

Área de estudo - O estudo foi conduzido no Parque Nacional

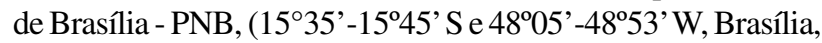
Distrito Federal) que conta com um área total de 30.556 ha. $\mathrm{O}$ PNB está situado na região nuclear do cerrado, caracterizada por forte sazonalidade de chuvas e altas temperaturas de ar, classificado como Aw (clima megatérmico com temperatura média do mês mais frio superior a $18^{\circ} \mathrm{C}$ e chuvas de verão) de acordo com o sistema de Köppen. A média anual de precipitação na região fica em torno de $1.500 \mathrm{~mm}$, variando de 750 a $2.000 \mathrm{~mm}$, praticamente concentrada nos meses de outubro a março (Adámoli et al. 1987). No ano de 2003, a precipitação anual foi de $1.394,5$ mm enquanto em 2004 foi de $1.753,2 \mathrm{~mm}$

Duas comunidades de cerrado típico com diferentes densidades de cobertura de lenhosas, distantes $10 \mathrm{~km}$ uma da outra, foram selecionadas. O cerrado típico é uma formação savânica composta por arbustos e árvores decíduas e sempreverdes (cobertura entre $20 \%$ e $50 \%$ ) dispersas sobre uma camada graminosa (Ribeiro \& Walter 1998). Essas comunidades foram incluídas no estudo de Ferreira et al. (2003) sobre variações sazonais dos índices espectrais da vegetação, onde dados estruturais da vegetação foram levantados a cada $1 \mathrm{~m}$ ao longo de transectos de $250 \mathrm{~m}$ (tabela 1). Aárea de cerrado mais densa apresentou cobertura lenhosa de $45,4 \%$ e será designada no presente trabalho por cerrado fechado (CF) enquanto a outra área, menos densa, apresentou cobertura lenhosa de $29,7 \%$ e será designada por cerrado aberto (CA).

Espécies estudadas - Foi realizado um inventário da vegetação pelo método ponto-quadrante nos dois sítios de estudo para comparar a composição das espécies arbustivoarbóreas. Foram selecionadas as espécies: Qualea parviflora Mart., Byrsonima coccolobifolia H. B. \& K., Roupala montana Aubl., Sclerolobium paniculatum Vog. e Kielmeyera coriacea (Spreng.) Mart., comuns aos dois sítios e com maiores valores de IVI (Índice de Valor de Importância, que consiste na soma da densidade, dominância e freqüência relativas). A monocotiledônea Vellozia squamata Pohl (=Vellozia flavicans Mart. ex Schult.) foi incluída por ser

Tabela 1. Dados estruturais da vegetação nos sítios de cerrado fechado (CF) e cerrado aberto (CA) do Parque Nacional de Brasília, Brasília-DF segundo Ferreira et al. (2003).

Table 1. Structural characteristics of vegetation in the "closed" cerrado (cerrado fechado - CF) and "open" cerrado (cerrado aberto - CA) sites at the Parque Nacional de Brasília, Brasília-DF according to Ferreira et al. (2003).

\begin{tabular}{lcccc}
\hline & $\begin{array}{c}\text { Cobertura } \\
\text { arbustivo-arbórea } \\
(\%)\end{array}$ & $\begin{array}{c}\text { Espaçamento } \\
\text { médio entre árvores } \\
(\mathrm{m})\end{array}$ & $\begin{array}{c}\text { Cobertura } \\
\text { graminosa verde (\%) } \\
\text { estação seca }\end{array}$ & $\begin{array}{c}\text { Cobertura } \\
\text { graminosa verde }(\%) \\
\text { estação chuvosa }\end{array}$ \\
\hline $\mathrm{CA}$ & 29,7 & 13,0 & 22,0 & 42,8 \\
$\mathrm{CF}$ & 45,4 & 7,3 & 14,5 & 37,9 \\
\hline
\end{tabular}


abundante e comum aos dois sítios. Essas espécies foram classificadas de acordo com Oliveira (2005) em decíduas (DC $=$ mais de duas semanas sem folhas: Q. parviflora, B. coccolobifolia e $K$. coriacea) e em sempre-verdes (SV = V. squamata, R. montana e $S$. paniculatum).

Análises foliares - Folhas expandidas localizadas no topo do dossel foram coletadas de cinco a seis indivíduos de cada espécie selecionada. As amostragens foram feitas a uma distância de pelo menos $10 \mathrm{~m}$ da estrada entre $10 \mathrm{~h} 00$ e $12 \mathrm{~h} 00$ da manhã. No campo, as folhas foram armazenadas em embalagens Tetrapak, protegidas da luz e mantidas sob refrigeração até o processamento em laboratório, realizado no mesmo dia da coleta.

Análises de pigmentos - As coletas para análise de pigmentos foliares foram realizadas em três períodos durante o ano de 2003: início do período seco (junho), auge da seca (setembro) e início do período chuvoso (novembro).

A extração de clorofila $a$ e de clorofila $b$ foi feita a partir de discos foliares de 5,5 mm de diâmetro, incubados em $1 \mathrm{~mL}$ de dimetilsulfóxido (DMSO) em banho-maria $\left(65^{\circ} \mathrm{C}\right)$, com quantificação por espectrofotometria UV-VIS (Richardson et al. 2002). O tempo de incubação necessário para a extração dos pigmentos foi determinado para cada espécie em testes preliminares. Os extratos foram transferidos para cubetas e os valores de absorbância $\left(A_{663}, A_{645}\right.$, para clorofilas $a$ e $b$, $A_{470}$ para carotenóides e $A_{532}$ e $A_{653}$ para antocianinas) foram lidos utilizando-se uma solução DMSO (branco) em espectrofotômetro da marca Genesys 2 PC (Thermo Spectronic). A estimativa das concentrações de clorofilas $a$ e $b$ foi feita a partir das seguintes equações: $\operatorname{cl} a\left(\mu \mathrm{g} \cdot \mathrm{mL}^{-1}\right)=12,7$ $\mathrm{A}_{663}-2,69 \mathrm{~A}_{645}, \mathrm{cl} b\left(\mu \mathrm{g} \cdot \mathrm{mL}^{-1}\right)=22,9 \mathrm{~A}_{645}-4,68 \mathrm{~A}_{663} \mathrm{e}$ $\mathrm{Tcl}=\mathrm{cl} a+\mathrm{cl} b$. A estimativa de carotenóides totais foi feita a partir dos mesmos extratos usados para as clorofilas, utilizando a equação de Lichtenthaler \& Wellburn (1983): Tcar $\left(\mu \mathrm{g} \cdot \mathrm{mL}^{-1}\right)=\left(1000 A_{470}-3,27 \mathrm{cl} a-104 \mathrm{cl} b\right) / 229$. As antocianinas foram extraídas a partir de outros discos retirados das mesmas folhas, incubados em $1 \mathrm{~mL}$ de metanol- $\mathrm{HCl}(0,1 \% \mathrm{HCl}, \mathrm{v} / \mathrm{v})$ a $-16{ }^{\circ} \mathrm{C}$ e mantidos a $4{ }^{\circ} \mathrm{C}$ no escuro por 24 horas. Os extratos foram transferidos para cubetas e a absorbância lida utilizando-se uma solução de metanol-HCl (branco). As concentrações de antocianina foram calculadas de acordo com as equações de Murray \& Hackett (1991) com correção do efeito da clorofila (AA) por meio da subtração de $24 \%$ da absorbância do comprimento de onda máximo da clorofila $\left(\mathrm{A}_{653}\right): \mathrm{AA}\left(\mu \mathrm{g} \cdot \mathrm{mL}^{-1}\right)=\mathrm{A}_{532}-0,24 \mathrm{~A}_{653}$.

Área foliar específica e concentrações de $\mathrm{N}$ e $\mathrm{P}-\mathrm{As}$ amostragens para determinação de área foliar específica (AFE) e concentrações de $\mathrm{N}$ e $\mathrm{P}$ foram realizadas em junho e novembro de 2004.

Para cálculo da área foliar específica (AFE), a área foliar foi determinada com o programa AREA(Caldas et al. 1992), cujo cálculo é feito a partir da imagem binária de uma folha. As imagens das folhas foram obtidas com um scanner de mesa.

As amostras de folhas foram secas em estufas a $70{ }^{\circ} \mathrm{C}$ até peso constante e então moídas. A concentração de $\mathrm{N}$ foi determinada de acordo com o procedimento Kjeldahl padrão após digestão em ácido sulfúrico. A determinação do fósforo foi feita a partir da espectrometria de emissão atômica com fonte de plasma (ICP), no Laboratório Campo (Paracatu, MG).

Análises estatísticas - O teste Kolmogorov-Smirnov foi utilizado para verificar se os dados apresentavam distribuição normal. Em caso negativo, a transformação logarítmica foi aplicada. Efeitos do sítio, fenologia e sazonalidade e suas interações sobre os parâmetros foliares foram testados através de uma análise multivariada com medidas repetidas no tempo (SPSS, vs10.0), onde o fator época do ano (mês) encontrava-se dentro do fator fenologia, e este dentro do fator sítio. As diferenças das concentrações de pigmentos entre os meses foram analisadas através do teste "post-hoc" de Tukey. Quando as análises demonstraram ausência de efeito significativo de um fator (sítio, fenologia, mês) sobre as concentrações de pigmentos foliares, N, P e AFE os valores foram agrupados, e somente os efeitos dos fatores significativos foram testados (tabelas 2, 3).

Tabela 2. Efeitos principais dos fatores sítio e período do ano (mês), e suas interações (MANOVAcom medidas repetidas, nível de $P \leq 0,05$ ), para todos os parâmetros foliares estudados em 2003: concentração de clorofila $a$ (Clor $a$ ), clorofila $b$ (Clor $b$ ), clorofila total (Clor total), carotenóides (Car) e antocianinas (Ant) e razão entres as clorofilas $a$ e $b$ (Clor $a / b)$ e clorofila total e carotenóides (Clor total/Car).

Table 2. Main effects of site and period of year (month) and their interactions using MANOVA for repeated measures (level of $P \leq 0.05$ ) regarding the foliar parameters studied in 2003: chlorophyll $a$ (Chlor $a$ ), chlorophyll $b$ (Chlor $b$ ), total chlorophyll (Total chlor), carotenoids (Car) and antocyanins (Ant) concentrations, chlorophylls $a$ and $b$ (Chlor $a / b)$ ratio and total chlorophyll and carotenoids ratio (Total chlor/Car).

\begin{tabular}{lcccccc}
\hline & Clor $a$ & Clor $b$ & Clor $a / b$ & Car & Ant & Clor total/Car \\
\hline Sítio & 0,037 & 0,797 & 0,931 & 0,399 & 0,012 & 0,633 \\
Mês & 0,000 & 0,000 & 0,083 & 0,004 & 0,000 & 0,051 \\
Sítio $\times$ Mês & 0,131 & 0,008 & 0,029 & 0,413 & 0,743 & 0,065 \\
\hline
\end{tabular}


Tabela 3. Efeitos principais dos fatores fenologia e período do ano (mês), e sua interação (MANOVA com medidas repetidas, nível de $P \leq 0,05)$, para todos os parâmetros foliares estudados em 2004: concentrações de nitrogênio (N) e de fósforo $(\mathrm{P})$, área foliar específica (AFE) e razão N:P.

Table 3. Main effects of phenology and period of year (month) and their interaction using MANOVA for repeated measures (level of $P \leq 0.05$ ) regarding the foliar parameters studied in 2004: nitrogen (N) and phosphorus (P) concentrations, specific leaf area (SLA) and N:P ratio.

\begin{tabular}{lcccc}
\hline & $\mathrm{N}$ & $\mathrm{P}$ & AFE & $\mathrm{N}: \mathrm{P}$ \\
\hline Fenologia & 0,000 & 0,000 & 0,000 & 0,740 \\
Mês & 0,000 & 0,000 & 0,227 & 0,000 \\
Fenologia $\times$ Mês & 0,005 & 0,097 & 0,005 & 0,591 \\
\hline
\end{tabular}

\section{Resultados e Discussão}

Variações nas concentrações de pigmentos foliares - A concentração dos pigmentos analisados foi significativamente influenciada pelos fatores "sítio" e "mês" bem como pela interação dos mesmos, mas não houve efeito significativo da "fenologia". A ausência de diferenças significativas nas concentrações médias de pigmentos entre espécies decíduas e sempre-verdes pode estar relacionada ao fato de que as diferenças nos padrões de produção e perda de folhas em grupos fenológicos de cerrado não sejam tão acentuadas como em outros sistemas sazonais (Oliveira 2005). Gamon et al. (1997) também não encontraram diferenças significativas para pigmentos foliares ao comparar espécies anuais, perenes decíduas e perenes sempreverdes, cultivadas em estufa.

Dessa forma, os dados de decíduas e sempreverdes foram agrupados e as diferenças testadas para sítio (cerrado fechado $\times$ cerrado aberto) e mês (junho, setembro e novembro) (tabela 2). No presente estudo, os maiores valores de concentração de clorofila $b$ foram encontrados no início (junho) em relação ao final da seca (setembro) nas duas áreas (figura 1). É também no início da estação seca, quando as folhas estão maduras mas a senescência não é acentuada, que se encontram diferenças significativas entre os sítios, com maiores concentrações de clorofilas $a$ e $b$ e menor razão clorofila $a / b$ na área mais densa. Em junho, o cerrado fechado apresentou valores cerca de $14 \%$ mais elevados de clorofila $a$ e $26 \%$ de clorofila $b$ em comparação com o cerrado aberto. Por outro lado, a razão das clorofilas $a / b$ foi $10 \%$ menor no cerrado fechado.
Em geral, as clorofilas $a$ e $b$ ocorrem em razão aproximada de 3 . As condições de crescimento e os fatores ambientais podem afetar essa razão. Plantas de sol ou expostas a condições de alta luminosidade apresentam razões de 3,2 a 4,0 enquanto nas plantas de sombra as razões variam de 2,5 a 2,9 (Lichtenthaler 1987) pois, em situações de baixa densidade de fluxo de fótons fotossinteticamente ativos ou baixas intensidades luminosas, o aumento de clorofila $b$ garante uma eficiência maior na absorção de energia (Lüttge 1997). Os valores da razão clorofila $a / b$ (entre 3 e 4) obtidos nos dois sítios no presente estudo indicam ambientes com alta luminosidade. Entretanto, a diferença observada no início da seca entre os sítios indica que a variação de cobertura entre as áreas é suficiente para ser refletida em maiores valores de clorofila $b$ na área mais densa, antes do período de intensa senescência (setembro).

A razão clorofila total/carotenóides também foi significativamente maior no cerrado fechado em relação ao aberto (aumento de 16\%) no início da seca (junho) devido às maiores concentrações de clorofilas no primeiro sítio, uma vez que não foram encontradas diferenças significativas para a concentração de carotenóides entre os sítios (figura 1). A detecção relativa dos pigmentos pode proporcionar informações a respeito do estado fisiológico das folhas (Sims \& Gamon 2002). Nos dois sítios (cerrado aberto e fechado) houve variação sazonal das concentrações de pigmentos relacionada à progressão da senescência foliar normalmente observada para comunidades de cerrado no final da estação seca (Nardoto et al. 2006). Em relação ao cerrado aberto, observou-se uma redução significativa das concentrações foliares das clorofilas $a$ e $b$, da razão entre elas, e dos carotenóides totais do início para o fim da estação seca. Na estação chuvosa (novembro), as concentrações dos pigmentos tenderam a aumentar, sendo significativamente maiores que em setembro para as clorofilas $a$ e $b$ e para a razão clorofila/ carotenóides. Redução das concentrações das clorofilas $a$ e $b$ e da razão clorofila/carotenóides (cerca de $29 \%$, $18 \%$ e $23 \%$, respectivamente) no fim em relação ao início da estação seca também foi observada no cerrado fechado. Entretanto, com exceção da razão clorofila/ carotenóides, não houve um aumento dos valores dos pigmentos no mês de novembro (estação chuvosa) como ocorreu no cerrado aberto.

O aumento subseqüente da concentração de clorofilas na estação chuvosa está relacionado à presença de folhas de uma nova cohorte. No cerrado fechado o aumento das concentrações de clorofilas 


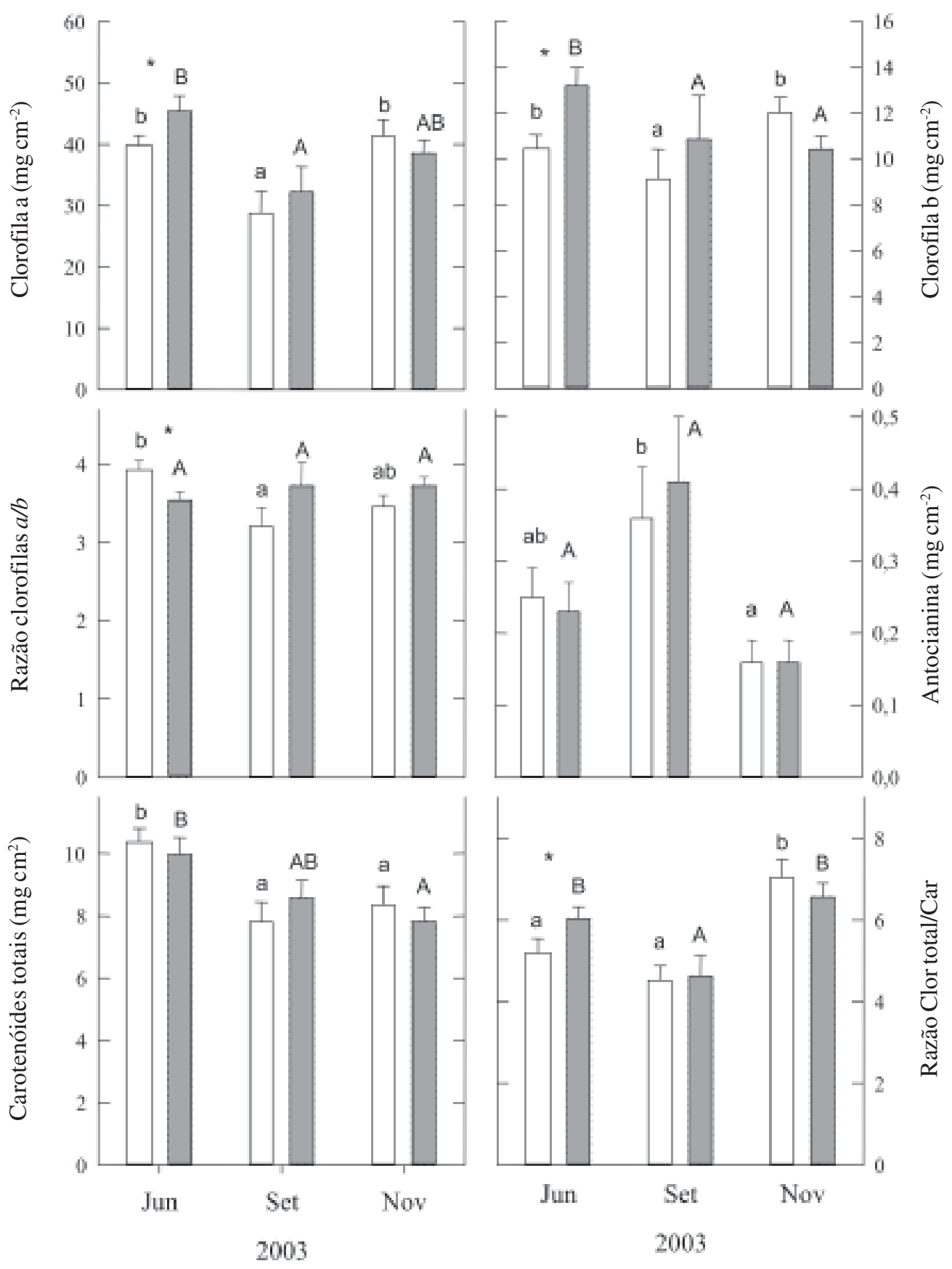

Figura 1. Concentrações médias \pm erro padrão dos pigmentos clorofilas $a, b$, carotenóides, razão clorofila $a /$ clorofila $b$ e razão clorofila total/carotenóides em folhas de seis espécies coletadas em duas áreas de cerrado senso restrito denominadas $\square$ = cerrado aberto $\square$ = cerrado fechado, nos períodos de junho, setembro e dezembro de 2003 no Parque Nacional de Brasília, DF. * indica diferenças significativas $(P \leq 0,05)$ entre as áreas em um mesmo período (mês). As letras diferentes indicam diferenças significativas $(P \leq 0,05)$ entre os períodos em um mesmo sítio (letras minúsculas para o cerrado aberto, e maiúsculas para o cerrado fechado).

Figure 1. Concentration means \pm standard error of the pigments chlorophylls $a, b$, carotenoids, chlorophyll $a$ /chlorophyll $b$ ratio and total chlorophyll/carotenoids ratio) of leaves of six woody species collected in two cerrado stricto sensu areas identified as $\square=$ open cerrado and $\square=$ closed cerrado in June, September and November of 2003 at Parque Nacional de Brasília, Brasília, DF. * indicates significant differences $(P \leq 0,05)$ between sites considering the same period (month). Different letters indicate significant differences $(P \leq 0,05)$ among periods within the same site. Lower case was used to open cerrado and upper case to closed cerrado. 
com a estação chuvosa pode ocorrer mais tardiamente, e por isso, não foi detectado neste estudo, sugerindo que uma frequiência maior de amostragem pode ser necessária para comparar comunidades de cerrado com diferentes estruturas. Em um estudo com espécies arbóreas tropicais, Cai et al. (2005) observaram que a concentração de clorofilas foi baixa nas folhas jovens, somente atingindo o seu valor máximo muitos dias após o término da expansão foliar. Houve também uma redução significativa da concentração de carotenóides no cerrado aberto do início da seca (junho) em relação aos períodos subseqüentes (setembro e novembro). Este padrão é semelhante ao encontrado para as clorofilas embora a concentração de carotenóides não retorne em novembro aos valores medidos nas folhas maduras (junho). Isto sugere um acúmulo de carotenóides com o amadurecimento da folha. A redução da razão clorofila total/carotenóides no cerrado fechado entre junho e setembro pode estar relacionada à tendência de declínio mais rápido das clorofilas em relação aos carotenóides quando as plantas estão sob condições de estresse ou durante a fase de senescência da folha. A retenção dos carotenóides vem sendo sugerida como mecanismo de foto-proteção durante o processo de senescência foliar (Sims \& Gamon 2002) e de dissipação do excesso de energia no sistema fotossintético (Demming-Adams \& Adams 1996), através do ciclo da xantofila.

Não foram encontradas diferenças significativas entre os sítios com relação às concentrações de antocianinas (figura 1). A concentração média de antocianinas também tendeu a aumentar no fim da seca (setembro). Gitelson et al. (2001) relatam que, embora, a concentração de antocianinas tenda a ser alta em folhas jovens, folhas de plantas sob diferentes estresses e folhas senescentes de algumas espécies também podem apresentar concentrações mais elevadas de antocianinas. Antocianinas podem estar envolvidas na proteção das folhas contra o excesso de luz, ou da radiação UV (Sims \& Gamon 2002) e contra predação, devido ao seu sabor desagradável. Dada a maior variabilidade da concentração de antocianinas entre as espécies em comparação com outros pigmentos, próximos estudos devem incluir um número maior de espécies que permita o agrupamento de espécies por faixas de concentração deste pigmento para evidenciar melhor possíveis diferenças ecológicas.

Variações na AFE e concentrações foliares de $\mathrm{N}$ e $\mathrm{P}$ As concentrações de $\mathrm{N}$ e $\mathrm{P}$, e AFE foram significativamente influenciadas pelos fatores "fenologia" e "mês" bem como pela interação dos mesmos para a concentração de N e AFE. Entretanto, não houve efeito significativo do sítio e dessa forma, os dados de cerrado fechado e aberto foram agrupados e as diferenças testadas para fenologia (decíduas e sempre-verdes) e mês (junho e novembro) (tabela 3).

Espécies decíduas apresentaram médias maiores de AFE em relação às sempre-verdes sendo $22 \%$ maiores em junho e $36 \%$ em novembro (figura 2). Não houve efeito significativo da sazonalidade sobre a área foliar específica (tabela 3). AFE é freqüentemente citada como característica chave ligada ao funcionamento das plantas (Reich et al. 1992, Wilson et al. 1999, Garnier et al. 2001) e baixos valores de AFE parecem ser vantajosos em condições de escassez de recursos ou fatores limitantes (Huante et al. 1995, Poorter \& Garnier 1999). Eamus et al. (1999) também encontraram maiores valores de AFE em espécies arbóreas sempre-verdes que em decíduas de savanas australianas.

Concentrações mais elevadas de $\mathrm{N}$ ( $47 \%$ em junho e $53 \%$ em novembro) e P (45\% em junho e $64 \%$ em novembro) foram encontradas em folhas das espécies sempre-verdes em relação às decíduas. As folhas das espécies dos dois grupos fenológicos coletadas na estação chuvosa (novembro) apresentaram maiores concentrações de $\mathrm{N}$ e $\mathrm{P}$, assim como maiores razões entre esses dois nutrientes, em relação às folhas coletadas na estação seca (junho). Esses resultados diferem dos obtidos por Franco et al. (2005) que encontraram maiores concentrações de nutrientes nas decíduas. Esta aparente discrepância pode estar relacionada à escolha das espécies, pois, no presente trabalho, o grupo das sempre-verdes incluiu uma leguminosa e o grupo das decíduas, não. No caso de Franco et al. (2005), há duas leguminosas entre as decíduas/brevidecíduas. Dentre as espécies de cerrado estudadas, as leguminosas são as que apresentam maiores concentrações de $\mathrm{N}$ foliar (dados não publicados) tanto em espécies sempre-verdes como decíduas. Assim, mesmo que a análise de grupos funcionais permita estudar mais facilmente estratégias ecológicas em ambientes com alta diversidade específica, a filogenia também deve ser considerada (Hoffman \& Franco 2003). Prior et al. (2003) compararam atributos foliares em quatro habitats sazonais na Austrália e destacaram que diferenças entre os habitats estão relacionadas à fenologia foliar e também à filogenia.

As análises de correlação indicam forte relação inversa entre AFE e os nutrientes $\mathrm{N}$ e $\mathrm{P}$ (tabela 4) tanto no início da seca como na estação chuvosa. Entretanto, 
outros estudos indicaram correlação positiva entre AFE e nitrogênio e fósforo foliar (Reich \& Walters 1994, Niinemets et al. 2001, 2002). Relações positivas podem ser parcialmente explicadas por uma fração maior de estruturas de suporte em folhas mais espessas e mais densas que possuem menor AFE e de acordo com uma menor necessidade de nutrição mineral para a construção de folhas com baixo AFE (Reich et al. 1992, Niinemets 1999). No entanto, a relação entre AFE e N pode ser diferente em comunidades onde a disponibilidade de água é variável devido a modificações nos investimentos em suporte em resposta a alterações na disponibilidade de água (Wrigth et al. 2001, Niinemets 2001). Ainda, dependendo do suprimento de nutrientes do solo, a concentração foliar de nutrientes pode variar amplamente para mesmos valores de AFE (e.g., Thompson et al. 1992), pois nem todo $\mathrm{N}$ e $\mathrm{P}$ foliares estão associados com as funções metabólicas das células.

O nitrogênio é geralmente considerado o nutriente mais limitante nos ecossistemas terrestres, mas em comunidades de solos ácidos, como o do cerrado, o fósforo pode ser o elemento limitante primário devido à oclusão de P por óxidos de ferro e alumínio (Vitousek \& Sanford Junior 1986, Reich et al. 1995). Nas espécies de cerrado estudadas, a razão N:P não diferiu significativamente entre sempre-verdes e decíduas, mas apresentou variação sazonal (tabela 3). Os valores

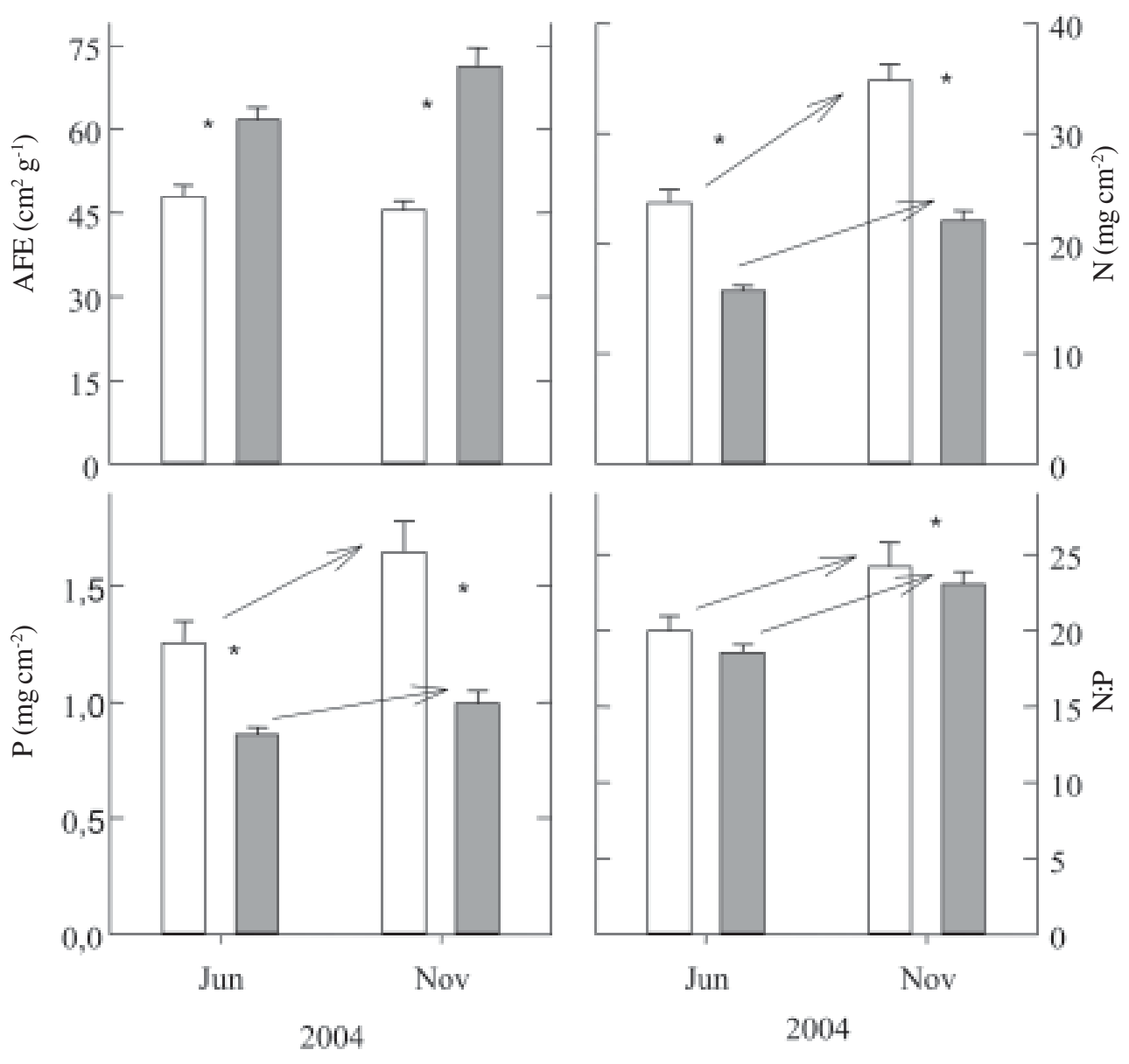

Figura 2. Área foliar específica (AFE) e concentrações médias \pm erro padrão de $\mathrm{N}$ e P e a razão N/P em folhas de espécies lenhosas ( $\square=$ sempre verdes e $\square=$ decíduas) coletadas em áreas de cerrado senso restrito nos períodos de junho e novembro de 2004 no Parque Nacional de Brasília, Brasília, DF. * indica diferenças significativas $(P \leq 0,05)$ entre os grupos fenológicos em um mesmo período (mês). As setas indicam diferenças significativas $(P \leq 0,05)$ entre os períodos em um mesmo grupo fenológico.

Figure 2. Specific Leaf Area (SLA) and concentration means \pm standard error of $N$ and P of woody species leaves $(\square=$ evergreen and $\square=$ deciduous) collected in two cerrado stricto sensu areas in June, September and November of 2003 at Parque Nacional de Brasília, DF. * indicates significant differences $(P \leq 0,05)$ between sites considering the same period (month). Arrows indicate significant differences $(P \leq 0,05)$ among periods within the same site. 
Tabela 4. Coeficientes de correlação e probabilidades (entre parênteses) entre AFE, $\mathrm{N}$ e $\mathrm{P}$ testadas separadamente por períodos (junho e novembro de 2004) e para os dois meses conjuntamente.

Table 4. Correlation coefficients and probabilities (parenthesis) between SLA, N and P tested separately (June and November of 2004) and grouped.

\begin{tabular}{lrrc}
\hline & Junho & Novembro & Junho + Novembro \\
\hline AFE $\times$ N & $-0,718(0,000)$ & $-0,823(0,000)$ & $-0,610(0,000)$ \\
AFE $\times$ P & $-0,569(0,000)$ & $-0,629(0,000)$ & $-0,573(0,000)$ \\
$\mathrm{N} \times \mathrm{P}$ & $0,778(0,000)$ & $0,784(0,000)$ & $0,762(0,000)$ \\
\hline
\end{tabular}

médios da razão N:P variaram entre 19,4 $\pm 4,4$ em junho e 24,3 \pm 7,6 em novembro, indicando uma limitação por P (Aerts \& Chapin 2000). As concentrações foliares de $\mathrm{N}$ e $\mathrm{P}$ apresentaram correlação positiva tanto no início da seca como na estação chuvosa (tabela 4) como observado para Nardoto et al. (2006) em folhas de 10 espécies de cerrado.

Os resultados mostraram que variações sazonais na química foliar de espécies arbustivo-arbóreas de Cerrado apresentam diferentes controles uma vez que as concentrações de nutrientes variaram em função da fenologia enquanto os pigmentos foliares, em especial as clorofilas, responderam às diferenças estruturais nos sítios.

Agradecimentos - Os autores agradecem José Salomão Silva, Mardocheu Pereira Rocha, Antônio Gumiero de Lima e Adriano Coelho Porto pela ajuda nos trabalhos de campo, Dra. Birgit Orthen pela orientação nos procedimentos de extração e quantificação de pigmentos, Dr. Augusto César Franco pelo uso de equipamentos do Laboratório de Fisiologia Vegetal, Prof. Dr. Lúcio Vivaldi pela orientação nas análises estatísticas e aos revisores anônimos por seus comentários que permitiram aprimorar o presente trabalho. Agradecimentos especiais são destinados à direção do Parque Nacional de Brasília pela colaboração na condução do presente estudo. O estudo foi financiado pelo "Experimento de Larga Escala Biosfera-Atmosfera na Amazônia" (LBA) através do Projeto LC-21 (Universidade de Brasília e Stanford University).

\section{Referências bibliográficas}

ADÁMOLI, J., MACÊDO, J., AZEVEDO, L.G. \& NETTO, J.M. 1987. Caracterização da região dos cerrados. In Solos dos cerrados: tecnologias e estratégias de manejo. (W.J. Goedert, ed.). Nobel, São Paulo, p.33-98.
AERTS, R. \& CHAPIN, F.S. III. 2000. The mineral nutrition of wild plants revisited: a re-evaluation of processes and patterns. Advances in Ecological Research 30:1-67.

CAI, Z-Q., SLOT, M. \& FAN, Z-X. 2005. Leaf development and photosynthetic proprieties of three tropical tree species with delayed greening. Photosynthetica 43:91-98.

CALDAS, L.S., BRAVO, C., PICCOLO, H. \& FARIA, C.R.S.M. 1992. Measurement of leaf area with a hand-scanner linked to a microcomputer. Revista Brasileira de Fisiologia Vegetal 4:17-20.

CRAINE, J.M., FROEHLE, J., TILMAN, D.C., WEDIN, D.A. \& CHAPIN, F.S. 2001. The relationships among root and leaf triats of 76 grassland species and relative abundance along fertility and disturbance gradients. Oikos 93:274-285.

DEMMING-ADAMS, B. \& ADAMS III, W.W. 1996. The role of xanthophyll cycle carotenoids in the protection of photosynthesis. Trends in Plant Science 1:21-26.

EAMUS, D., MYERS, B., DUFF, G. \& WILLIAMS, R. 1999. Seasonal changes in photosynthesis of eight savanna tree species. Tree Physiology 19:665-671.

FERREIRA, L.G., YOSHIOKA, H., HUETE, A. \& SANO, E.E. 2003. Seasonal landscape and spectral vegetation index dynamics in the Brazilian Cerrado: An analysis within the Large-Scale Biosphere-Atmosphere Experiment in Amazônia (LBA). Remote Sensing of Environment 87:534-550.

FRANCO, A.C., BUSTAMANTE, M.M., CALDAS, L.S., GOLDSTEIN, G., MEINZER, F.C., KOZOVITS, A.R., RUNDEL, P. \& CORADIN, V.T.R. 2005. Leaf functional traits of Neotropical savanna trees in relation to seasonal water deficit. Trees - Structure and Function 19:326-335.

GAMON, J.A., SERRANO, L. \& SURFUS, J.S. 1997. The photochemical reflectance index: an optical indicator of photosynthetic radiation use efficiency across species, functional types, and nutrient levels. Oecologia 112:492-501.

GARNIER, E., LAURENT, G., BELLMANN, A., DEBAIN, S., BERTHELIER, P., DUCOUT, B., ROUMET, C. \& NAVAS, M.L. 2001. Consistency of species ranking based on functional leaf traits. New Phytologist 152:69-83.

GITELSON,A.A., MERZLYAK, M.N. \& CHIVKUNOVA, O.B. 2001. Optical properties and non-destructive estimation of anthocyanin content in plant leaves. Photochemistry and Photobiology 74:38-45.

GRIME, J.P., THOMPSON, K., HUNT, R., HODGSON, J.G., CORNELISSEN, J.H.C., RORISON, I.H., HENDRY, GA.F., ASHEDEN, T.W., ASKEN, A.P., BAND, S.R., BOSSARD, C.C., CAMPBELL, B.D., COOPER, J.E.L., DAVISON, A.W., GUPTA, P.L., HALL, W., HAND, D.W., HANNAH, M.A., HILLIER, S.H., HODKINSON, D.J., JALILI, A., LIU, Z., MACKEY, N., MATTHEWS, N., MOWFORTH, M.A., NEAL, A.M. READER, R.J., REILING, K., ROSSFRASER, W., SPENCER, P.E., SUTTON, F., TASKER, D.E., THORPE, P.C. \& WHITEHOUSE, J. 1997. Integrated screening validates primary axes of specialization in plants. Oikos 79:259-281. 
HOFFMANN, W.A. \& FRANCO, A.C. 2003. Comparative growth analysis of tropical savanna and forest trees using phylogenetically-independent contrasts. Journal of Ecology 91:475-484.

HUANTE, P., RINCÓN, E. \& ACOSTA, I. 1995. Nutrient availability and growth rate of 34 woody species from a tropical deciduous forest in Mexico. Functional Ecology 9:49-858.

LICHTENTHALER, H.K. 1987. Chlorophyll and carotenoids: pigments of photosynthetic biomembranes. Methods in Enzymology 148:331-382.

LICHTENTHALER, H.K. \& WELLBURN, A. 1983. Determinations of total carotenoids and chlorophylls a and $b$ of leaf extracts in different solvents. Biochemical Society Transactions 603:591-592.

LÜTTGE, U. 1997. Physiological ecology of tropical plants. Springer.

MURRAY, J.R. \& HACKETT, W.P. 1991. Difydroflavonol reductase activity in relation to differential anthocyanin accumulation in juvenile and mature phase Hedera helix L. Plant Physiology 97:343-351.

NARDOTO, G.B., BUSTAMANTE, M.M.C., PINTO, A.S., KLINK, C.A. 2006. Nutrient use efficiency at ecosystem and species level in savanna areas of Central Brazil and impacts of fire. Journal of Tropical Ecology 22:1-11.

NIINEMETS, Ü. 1999. Components of leaf dry mass per area - thickness and density - alter leaf photosynthetic capacity in reverse directions in woody plants. New Phytologist 144:35-47.

NIINEMETS, Ü. 2001. Global-scale climatic controls of leaf dry mass per area, density, and thickness in trees and shrubs. Ecology 82:453-469.

NIINEMETS, Ü., ELLSWORTH, D.S., LUKJANOVA, A. \& TOBIAS, M. 2001. Site fertility and the morphological and photosynthetic acclimation of Pinus sylvestris needles to light. Tree Physiology 21:1231-1244.

NIINEMETS, Ü., PORTSMUTH, A. \& TRUUS, L. 2002. Leaf structure and photosynthetic characteristics, and biomass allocation to foliage in relation to foliar nitrogen content and tree size in three Betula species. Annals of Botany 89:191-204.

OLIVEIRA, E.L. 2005. Fenologia, demografia foliar e características foliares de espécies lenhosas em um Cerrado sentido restrito no Distrito Federal e suas relações com as condições climáticas. Tese de doutorado, Universidade de Brasília, Brasília.

POORTER, H. \& GARNIER, E. 1999. Ecological significance of inherent variation in relative growth rate and its components. In Handbook of functional plant ecology (F.I. Pugnaire \& F. Valladares, eds.). Marcel Dekker, Inc., New York p.81-120.
PRIOR, L.D., EAMUS, D. \& BOWMAN, D.M.J.S. 2003. Leaf attributes in the seasonally dry tropics: a comparison of four habitats in northern Australia. Functional Ecology 17:504-515.

PRIOR, L.D., BOWMAN D.M.J.S. \& EAMUS, D. 2004. Seasonal differences in leaf attributes in Australian tropical tree species: family and habitat. Functional Ecology 18:707-718.

REICH, P.B. \& WALTERS, M.B. 1994. Photosynthesisnitrogen relations in Amazonian tree species. II. Variation in nitrogen vis-a-vis specific leaf area influences massand area-based expressions. Oecologia 97:73-81.

REICH, P.B., WALTERS, M.B. \& ELLSWORTH, D.S. 1992. Leaf lifespan in relation to leaf, plant, and stand characteristics among diverse ecosystems. Ecological Monographs 62:365-392.

REICH, P.B., ELLSWORTH, D.S. \& UHL, C. 1995. Leaf carbon and nutrient assimilation and conservation in species of differing successional status in an oligotrophic Amazonian forest. Functional Ecology 9:65-76.

RIBEIRO, J.F. \& WALTER, B.M.T. 1998. Fitofisionomias do bioma cerrado. In Cerrado: ambiente e flora (S.M. Sano \& S.P. Almeida, eds.). Embrapa-CPAC, Planaltina, p.89-166.

RICHARDSON, A.D., DUIGAN, S.P. \& BERLYN, G.P. 2002. An evaluation of noninvasive methods to estimate foliar chlorophyll content. New Phytologist 153:185-194.

SIMS, D.A. \& GAMON, J.A. 2002. Relationships between leaf pigment content and spectral reflectance across a wide range of species, leaf structures and developmental stages. Remote Sensing of Environment 81:337-354.

THOMPSON, W.A., HUANG, L.K. \& KRIEDEMANN, P.E. 1992. Photosynthetic response to light and nutrients in sun-tolerant and shade-tolerant rainforest trees. II. Leaf gas exchange and component processes of photosynthesis. Australian Journal of Plant Physiology 19:19-42.

VITOUSEK, P.M. \& SANFORD JUNIOR, R.L. 1986. Nutrient cycling in moist tropical forest. Annual Review of Ecology and Systematics 17:137-167.

WILSON, P.J., THOMPSON, K. \& HODGSON, J.G. 1999. Specific leaf area and leaf dry matter content as alternative predictors of plant strategies. New Phytologist 143:155-162.

WRIGHT, I.J., REICH, P.B. \& WESTOBY, M. 2001. Strategy shifts in leaf physiology, structure and nutrient content between species of high- and lowrainfall and high- and low-nutrient habitats. Functional Ecology 15:423-434. 